\title{
P8 Professionalisierung durch Organisation und Akademisierung
}

\author{
Tanja Dreischer
}

Online publiziert: 30. November 2011

(C) Springer-Verlag 2011

Hintergrund und Methode: Über die Praxis und Entwicklung von professioneller Krankenpflege in Irland lässt sich in Deutschland nur wenig Literatur finden - völlig zu Unrecht, wie sich bei einem vierwöchigen, von der Robert-BoschStiftung im Rahmen des Internationalen Hospitationsprogrammes Pflege und Gesundheit finanzierten Aufenthalts zeigen sollte. Im Rahmen der Hospitation sollte u. a. eine Frage darauf gefunden werden, wie es der professionellen Krankenpflege gelingen konnte, einen stärkeren Einfluss auf das Gesundheitssystem zu erkämpfen und die eigene Professionalisierung entscheidend voranzubringen.

Ergebnisse: Im Jahr 1998 wurde von der Regierung eine Studie in Auftrag gegeben, welche aufzeigen sollte, wie einer Stagnation der Entwicklung der Krankenpflege entgegengesteuert werden könnte. Der daraus resultierende Report of Comission on Nursing zeigte, dass es zur weiteren Professionalisierung der Krankenpflege unterschiedlicher Verbesserungen bedurfte: Pflegefachkräfte benötigen eine höherqualifizierte Ausbildung, andere Möglichkeiten der Weiterbildung sowie mehr Verantwortung und Autorität. Zu diesem Zweck wurde die dreijährige Ausbildung an Krankenpflegeschulen zugunsten eines universitären Studiums ersetzt. Dieser fachlichen Höherqualifizierung sollten mehr Autonomie und Verantwortung sowie die finanzielle Anerkennung folgen.

Den Pflegefachkräften ging all dies allerdings nicht zügig genug, so dass es kurze Zeit nach der Veröffentlichung des Reports zu einem flächendeckenden Streik des Krankenpflegepersonals in Irland kam. Ein solcher - im deutschen Kontext kaum vorstellbarer - Ausstand war möglich, da
$98 \%$ der Pflegefachkräfte in Berufsunionen vereinigt sind und folglich über ein hohes Machtpotential verfügen.

Infolgedessen sah sich die Regierung gezwungen, der Krankenpflege mehr Mitsprache in der Gesundheitspolitik einzuräumen. Im Ergebnis entstand das dem Gesundheitsministerium angehörende Health Service Executive (HSE), deren Vorsitzende, Director of Nursing, als Beraterin des Gesundheitsministers fungiert. Parallel $\mathrm{zu}$ dieser neuen Struktur wurde ein von der Regierung finanziertes National Council for the Professional Development of Nursing and Midwifery geschaffen. Dessen Hauptaufgabe besteht darin, die einheitlichen Richtlinien für die Qualifikationen, beispielsweise der Clinical Nurse Specialists zu gewährleisten und deren Anerkennung transparent zu gestalten.

Im Zuge der Akademisierung entstand bei den Pflegefachkräften ein beeindruckender Wille zur Weiterqualifizierung, der die Professionalität im täglichen Handeln entscheidend steigerte.

Schlußfolgerung: Dies sind nur zwei Prozesse, die aufzeigen können, wie es der Krankenpflege gelang, Engagement, Selbstbewusstsein und Einfluss der Krankenpflege $\mathrm{zu}$ fördern: durch Akademisierung und hohen Organisationsgrad. Beides ist sicherlich auch in Deutschland nützlich, um den Stellenwert der Krankenpflege mittelfristig zu verbessern.

T. Dreischer $(\bowtie)$

Carus Akademie am Universitätsklinikum Carl-Gustav-Carus

der TU Dresden, Dresden, Deutschland 